Effi ci ent ul trashort-pul se gener at $i$ on of Yb: YAG I aser over coming the fI uor escence spectruml i mit by usi ng nonl i near medi um

\begin{tabular}{|l|l|}
\hline $\begin{array}{l}\text { j our nal or } \\
\text { publ i cat } \mathrm{i} \text { on } \mathrm{ti} \text { t l e }\end{array}$ & 2008 I EEE/LEOS ANNUAL MEETI NG \\
\hline page range & ThS2- 1- ThS2- 2 \\
\hline year & 2008 - 11 \\
\hline URL & ht t p: //hdl . handl e. net /10098/6951 \\
\hline
\end{tabular}




\title{
Efficient ultrashort-pulse generation of Yb:YAG laser overcoming the fluorescence spectrum limit by using nonlinear medium
}

\author{
Shinichi MATSUBARA, Masaki TAKAMA, Masahiro INOUE, and Sakae KAWATO \\ Graduate School of Engineering, University of Fukui, 3-9-1 Bunkyo, Fukui, Fukui 910-8507, Japan \\ matubara@optele.fuee.fukui-u.ac.jp, kawato@fuee.fukui-u.ac.jp \\ Yuzo ISHIDA \\ The Institute of Physical and Chemical Research, Riken Wako Insutitute, 2-1 Hirosawa, Wako, Saitama, Japan.
}

\begin{abstract}
One-hundred-ten-fs and 72-fs pulse-widths were obtained directly from a mode-locked Yb:YAG laser with SESAM and without SESAM, respectively. The laser-spectrum-center and the fluorescence-center were almost same. The oscillation-spectra were much broader than the fluorescence.
\end{abstract}

In recent years, there has been great interest in mode-locked lasers with high average output powers, high efficiency, high reliability, and compactness. They have several applications in scientific and industrial fields such as wavelength conversion, nonlinear spectroscopy, and superfine material processing. To achieve high power short pulse laser, a broad emission spectrum and favorable thermal properties are important. $\mathrm{Yb}^{3+}$-ion doped materials are believed to be the most promising materials to satisfy those needs. $\mathrm{Yb}^{3+}$-ion has only two Stark manifolds, which reduces undesirable effects such as excited state absorption, cross-relaxation and quantum defect so that the thermal loads are reduced. Their broad spectra allow for directly diode-pumped high power short pulse lasers. Several kinds of diode-pumped $\mathrm{Yb}^{3+}$-ion doped laser generating sub-100 fs pulses as well as high power femtosecond lasers have been reported.

The $\mathrm{Yb}^{3+}$-ion doped crystals used to generate sub-100 fs pulses so far, however, have favorable thermal properties. In addition their extremely broad fluorescence spectrum tends to have a large overlap with the corresponding absorption spectrum, which leads to reabsorption loss. For example, monoclinic double tungstates and orthovanadates are good alternatives, and 100-fs and 120-fs pulses were produced with a SESAM for the laser materials of $\mathrm{Yb}: \mathrm{KY}\left(\mathrm{WO}_{4}\right)_{2}$ (abbreviated as $\mathrm{Yb}: \mathrm{KYW}$ ) and $\mathrm{Yb}: \mathrm{YVO}_{4}$, respectively. Sub-100-fs pulses were also obtained with a SESAM from an $\mathrm{Yb}: \mathrm{LuVO}_{4}$ laser as well as from an $\mathrm{Yb}$ :glass laser. Pulses as short as $47 \mathrm{fs}$ were

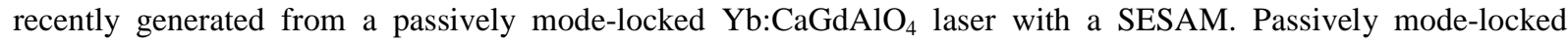
Yb:YAG lasers with a SESAM in low-output-power regions generated pulses as short as $340 \mathrm{fs}$ at $1030 \mathrm{~nm}$ and 136 fs at $1050 \mathrm{~nm}$, and in high-output-power regions a pulse energy as high as $5.1 \mu \mathrm{J}$ in 800 -fs pulses was achieved.

The pulse width of the all lasers was restricted to the limit of the fluorescence spectrum. In the case of Yb:YAG, at the gain center wavelength of $1030 \mathrm{~nm}$, the fluorescence spectral width is $9.5 \mathrm{~nm}$ in FWHM and the Fourier transform limited pulse width is $120 \mathrm{fs}$. Spectral broadening by filtering of the gain center was achieved previously. The pulse width of $100 \mathrm{fs}$ was observed and the laser center wavelength was shifted to be a low gain center of around $1050 \mathrm{~nm}$; however, for efficient laser oscillation, it is suitable that the center of the gain and the lasing is almost same. The shortest pulse width at the gain center was achieved previously to be around $300 \mathrm{fs}$ for hundreds $\mathrm{mW}$ output power regions and around $1 \mathrm{ps}$ for over tens $\mathrm{W}$ output power regions.

In this paper, efficient spectral broadening by nonlinear medium and the ultrashort-pulse generation overcoming the limit of the fluorescence spectrum are achieved. Around and below 100 fs pulse width at the gain center wavelength of $1030 \mathrm{~nm}$ were obtained directly from mode-locked Yb:YAG lasers pumped by a low-brightness, broad-area laser-diode with SESAM and without SESAM. A Yb:YAG microchip gain module was used for the short pulse mode-locked laser. Highly efficient $\mathrm{CW}$ laser operation was demonstrated by using the microchip gain module [1]. The Yb:YAG crystal (Scientific Material Corp.) was used with the ion density of 20 at.\% and $3 \mathrm{~mm} \mathrm{x}$ $3 \mathrm{~mm}$ size surface perpendicular to the $\langle 100\rangle$ axis with $1 \mathrm{~mm}$ thickness. The pump surface is used as the end mirror of the laser resonator and high-reflection coated between $1030 \mathrm{~nm}$ to $1080 \mathrm{~nm}$ laser wavelength and anti-reflection coated at $940 \mathrm{~nm}$ pump wavelength. Another surface of the crystal is antireflection coated for the pump and the laser wavelength. The Yb:YAG crystal is adhered to a $1 \mathrm{~mm}$ thickness Sapphire plate with antireflection coated on both surfaces and mounted to a copper heat-sink for cooling. The pump beam from a single emitter, low brightness wide area laser diode was focused $25 \mu \mathrm{m} \times 75 \mu \mathrm{m}$ spot diameter in the laser crystal. The LD wavelength was $942 \mathrm{~nm}$ and the absorption efficiency of the gain module was $80 \%$.

A nonlinear medium was used in the mode-locked laser oscillator to generate a broad emission spectrum caused by SPM. The laser beam in the cavity was strongly focused into the nonlinear medium. The pulse duration of $72 \mathrm{fs}$ was obtained from Kerr-lens mode-locked laser without SESAM and the autocorrelation trace is shown Fig.1(a). 
The center wavelength was $1030 \mathrm{~nm}$ and the spectral bandwidth was $17 \mathrm{~nm}$ [Fig.1(b)]. This broadening was considered to be caused in the strong self-phase modulation effect in the nonlinear material. The pulse repetition rate was $62 \mathrm{MHz}$. The average output power was $78 \mathrm{~mW}$ for the pump power of $2.5 \mathrm{~W}$. In our knowledge, the pulse duration is shortest for mode-locked Yb:YAG laser at center wavelength of $1030 \mathrm{~nm}$. Kerr-lens mode-locked Yb:YAG laser was realized by using a nonlinear medium in the laser cavity.

A pulse duration of 110 fs was achieved from the mode-locked Yb:YAG laser with SESAM and the autocorrelation trace is shown in Fig. 1 (a). The laser center wavelength and the spectral width were $1030 \mathrm{~nm}$ and 12 nm, respectively [Fig. 2(b)]. The average output power was $220 \mathrm{~mW}$ at a pump power of $1.6 \mathrm{~W}$. The optical-tooptical conversion efficiency is $14 \%$ for the incident pump power. In spite of a large insertion loss of SESAM and a low mode-matching efficiency between laser beam and an elliptical multimode pump-beam from the lowbrightness-laser-diode, is estimated to be around only $30 \%$, the value of the optical-to-optical conversion efficiency is high.

In conclusion, we have demonstrated femtosecond pulse generations from a directly diode pumped Yb:YAG laser overcoming the gain spectral limit by using a nonlinear medium in the laser cavity with and without SESAM. The shortest pulse duration of 72 fs was obtained from a Kerr-lens mode-locked Yb:YAG laser with an average power of $78 \mathrm{~mW}$ at $1030 \mathrm{~nm}$ center wavelength. The shortest pulse duration of $110 \mathrm{fs}$ was also generated from mode-locked Yb:YAG lasers with SESAM with a optical-to-optical conversation efficiency of 14\%. We believe that short pulse nearly $50 \mathrm{fs}$ and high average power over $1 \mathrm{~W}$ will be obtained in mode-locked Yb:YAG laser by the nonlinear medium and high power pumping.

References

[1] Matsubara, S., Ueda, T., Kawato, S., and Kobayashi, T., "Highly efficient laser oscillation of Yb:YAG at room temperature”, 2007, Jpn. J. App. Phys., 46, L132-L134.

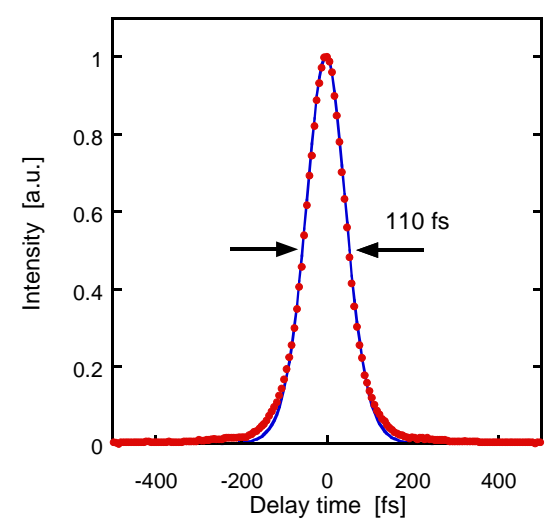

Fig.1(a). Autocorrelation trace of $72 \mathrm{fs}$ pulse for the Kerr-lens mode-locked Yb:YAG laser. The experimental data (circles) and $\operatorname{sech}^{2}$-fitting curve (solid curve) are shown.

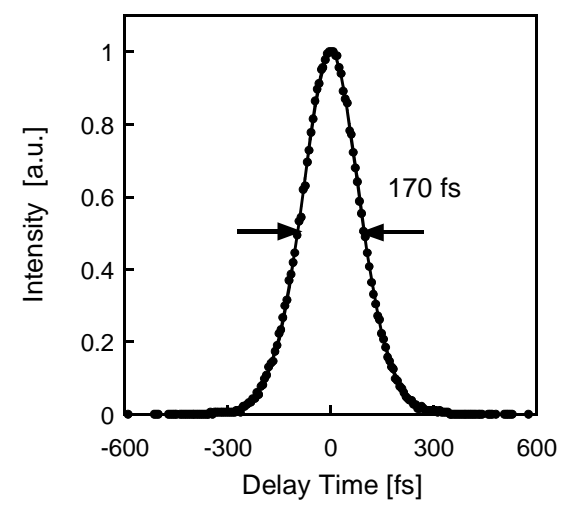

Fig. 2(a) Autocorrelation trace of the mode-locked Yb:YAG laser with SESAM. The experimental data (circles) and sech $^{2}$ fitting curve (solid curve) are shown. The pulse width was estimated to be $110 \mathrm{fs}$

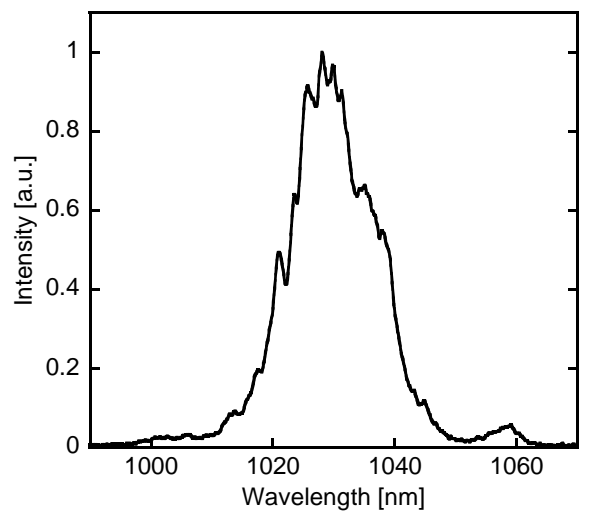

Fig.1(b). Spectrum of 72 fs pulse from the Kerr-lens modelocked Yb:YAG laser.

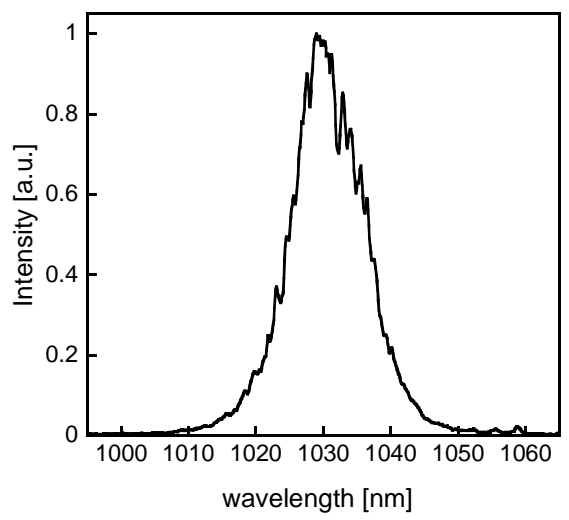

Fig. 2(b) Spectrum of the mode-locked Yb:YAG laser with SESAM. The spectral width was $12 \mathrm{~nm}$ in FWHM. 